\title{
Historique et évolution du dopage
}

\section{Historical review and evolution of doping}

Jean-Pierre de MONDENARD

Médecin du sport, Observateur de la lutte antidopage depuis le début des années 1970, il a publié

quatre ouvrages sur ce thème : Le dossier noir du dopage - éd. Hachette, 1981 ; Drogues et dopages - éd. Quel

Corps ?, 1987 ; Dictionnaire des substances et procédés dopants en pratique sportive - éd. Masson, 1996.

Centre de médecine, nutrition et traumatologie du sport, 12, avenue Georges 94430 CHENNEVIÈRES SUR MARNE - Té1 : 0145763835 - Fax : 0145940677

(Reçu le 13 décembre 1999 ; accepté le 12 janvier 2000)

\section{$R E ́ S U M E ́$}

L'histoire du sport recoupe continuellement celle du dopage. De même, la tentation du dopage ne lui est pas spécifique et elle a, plus largement, attiré l'homme vers la stimulation de toutes ses activités physiques et intellectuelles.

Dès l'origine, pour survivre, l'homme a été confronté aux réalités de la chasse et de la guerre. Il a directement perçu la nécessité d'être fort et, si possible, le plus fort, face à l'animal ou face au rival.

Plus près de nous, les Jeux olympiques antiques ont entamé la dérive de la préparation guerrière vers le sport. Ce dernier a ensuite poursuivi sa route dans les mentalités et à travers les siècles. Au fil de cette évolution, le problème ne s'est jamais posé en terme de "dopage", ni pour les hommes d'armes ni pour les sportsmen.

Pour ces champions, héritiers des guerriers et des chasseurs, la règle était simple. Peu importaient les moyens, l'essentiel étant d'être le meilleur. Et de ces moyens, l'histoire livre un abondant catalogue.

Tout au long de la préhistoire, ce sont les plantes - seules préparation naturelles disponibles - qui vont envahir les enceintes sportives.

A partir de la fin du $X I X^{\circ}$, la permanence du désir d'améliorer les possibilités humaines à n'importe quel prix se pérennise mais plus on avance dans le temps et plus les recettes deviennent sophistiquées. C'est la grande époque des breuvages magiques et potions accélératrices venant en droite ligne des hippodromes où l'on retrouve, pêle-mêle, atropine, caféine, strychnine, cocaïne, arsenic...

La seconde guerre mondiale va livrer à toute la planète spor-

\section{SUMMARY}

The story of sports follows constantly that of doping. The temptation of doping is not a specific part of the sport, it has more widely drawn the man to stimulation of all his intellectual and physical powers.

From the beginning and in order to survive, the man has had to face up the reality of hunting and war. He has directly perceived the need of being strong and, maybe the strongest, in front of the animal or a rival.

Close up on us, ancient olympic games had started the drift of war preparation toward the sports. This one has further followed its road into mentalities throughout centuries. During this evolution, the matter has never been laid in terms of "doping", neither for warriors nor sportmen.

For the champions, heirs of warriors and hunters, the rule was simple. No matter the means, the main being to be the best. The story gives an extensive list of these means.

During prehistory, the plants - being the only natural preparations available - were overrunning the sports arenas.

From the end of the XIX $X^{i h}$ century, the constant wish to enhance human possibilities is perpetuated, whatever the price to pay, so the more we wear on, the more the formulas are sophisticated. It is the era of magic beverages and speed up potions, directly imported from racecourses, including higgledy-piggledy : atropine, caffeine, strychnine, cocaine and arsenic...

Second World War will deliver to the sport planet, via the sky "sportmen", the amphetamines, a synthetic substance acting on the central nervous system. The big era of the alert amines will last in the beginning of the '70. Their decline will 
tive via les "athlètes" du ciel, les amphétamines, des substances synthétiques agissant sur le système nerveux central. La grande période des amines de l'éveil va durer jusqu'au début des années 70. Leur déclin surviendra avec la mise en place des premiers tests antidopage. Pendant les trente dernières années, l'histoire des substances dopantes va se décliner au rythme des performances des toxicologues qu'ils soient dans le camp des tricheurs ou dans celui de la lutte antidopage. Chaque bataille portera le nom d'une ou deux molécules : corticoïdes, stérö̈des anabolisants (1970), testostérone, hormone de croissance (1980), érythropoiétine (1990). On est passé progressivement des substances exclusivement artificielles n'ayant aucun équivalent endogène, à des produits mimant à la perfection hormones et précurseurs qui existent à l'état naturel dans l'organisme, ce qui renddans l'état actuel des connaissances - leur détection très aléatoire.

Aujourd'hui, le dopage prenant une place de plus en plus envahissante dans l'actualité sportive, il est probable que pour les décennies à venir, il faudra prévoir dans cette saga historique de nombreuses mises à jour.

\section{MOTS-CLÉS}

substances dopantes, lutte antidopage, amphétamines, corticoüdes, stéroïdes anabolisants, testostérone, hormone de croissance, érythropoïétine.

\section{Une très vieille pratique endémique en plein essor}

Dès l'origine, pour survivre, l'homme a été confronté aux réalités de la chasse et de la guerre. Il a directement perçu la nécessité d'être fort, et si possible, le plus fort face à l'animal ou face au rival. Très vite, l'éducation du corps a fait partie intégrante de la plupart des cultures anciennes à travers le monde, en Chine, en Inde et aux Amériques.

Plus près de nous, les Grecs lui ont donné une impulsion encore plus nette, trouvant son aboutissement dans la république belliqueuse de Sparte. Puis les Jeux olympiques, delphiques ou corinthiens ont entamé la dérive de la préparation guerrière vers le sport. Une évolution parallèle a entraîné ailleurs la naissance des Jeux celtiques. Le sport a ensuite poursuivi sa route dans les mentalités et à travers les siècles : choule, calcio, tournoi, paume, golf en ont formé les étapes jusqu'à l'ère britannique des "Paris", laquelle a précédé la symbolique rénovation des Jeux et l'essor du sport moderne. Au fil de cette évolution, le problème ne s'est jamais posé en terme de "dopage", pour les hommes d'armes ni pour les amateurs de paris. Pour ces champions, héritiers des guerriers et des chasseurs, la règle était simple. Peu importaient les moyens, l'essentiel étant d'être le meilleur. Et de ces moyens, l'histoire livre un abondant catalogue. take place with the first doping tests. During the last thirty years, the story of doping substances will go forward the rhythm of the performances of toxicologists, being in the cheaters side or in the antidoping side. Every fight will bear the name of one (or two) molecules : corticoides, anabolic steroids (1970), testosterone, growth hormone (1980), erythropoietin (1990). We pass progressively from an artificial substance with no endogenic equivalent to some products mimicing hormones and precursors, naturally existing in the body, which makes their detection very risky, taking into account the current knowledge.

Today, as doping is taking a bigger place in sports news, it should be important to foresee several updatings for that historical "saga", in the next decades.

\section{KEY-WORDS}

doping substances, antidoping fight, amphetamines, corticoids, anabolic steroids, testosterone, growth hormone, erythropoietin.

\section{La préhistoire}

En étudiant les sociétés primitives, les ethnologues n'ont pas manqué de remarquer la place importante faite au sang, à son rôle, à son pouvoir vivifiant et symbolique. Georges-Henri Luquet, dans La Pensée primitive, remarque :

"Le sang, principe de vie et de force, est le fortifiant par excellence. Se nourrir de chair qui est sang, c'est introduire en soit, assimiler une force étrangère. Peut-être faut-il voir là l'une des raisons qui ont amené l'homme à devenir carnivore, et parfois cannibale".

Sans doute cette idée a-t-elle conduit, dès le VIe siècle av. J.-C., les athlètes de l'Antiquité à tenter d'augmenter leur force physique par la consommation de viandes. Les chroniques indiquent qu'ils en dévoraient de grandes quantités, absorbant des viandes différentes selon leur spécialité : porc gras pour les lutteurs voulant développer leur masse corporelle, chèvre pour les sauteurs, taureau pour les pugilistes et les lanceurs.

Dans Vaincre à Olympie, Maurice Genevoix qui, pour écrire ce livre, s'est livré à de minutieuses recherches, fait dire à Menestrée, racontant l'histoire d'Euthymos, élève de Milon de Crotone :

"Faire ce qu'il faut, pour Euthymos, c'est obéir à Milon de Crotone, manger les mets qu'il a prescrits, les viandes grillées, les fromages frais, boire aux repas un cotyle de vin pur assaisonné d'un oignon cru. Pourquoi juste ce poids de viande, cette exacte mesure de vin et non pas moins ou davantage? Parce que l'expérience de Milon, ses années, ses souvenirs, ses victoires en ont décidé ainsi. Le grand 
Alipte a connu le temps où les athlètes ne se nourrissaient que de fromages égouttés, et leur teint devenait blafard et leurs veines charriaient du lait aigre ; jusqu'au jour où Dromeus de Stymphale, s'étant rassasié de bonne viande, distança ses rivaux dans le stade, seul coureur l'estomac plein, au sang rouge".

D'ailleurs, cette tendance à vouloir profiter des aptitudes sportives des animaux s'est pérennisée au cours des siècles. Ainsi, des laboratoires pharmaceutiques, afin de stimuler la vente de leurs médicaments, n'hésitent-ils pas à leur donner des noms particulièrement évocateurs ; c'est le cas d'un vieux remède contre l'essoufflement ayant comme nom de spécialité "Pulmo vulpis" (poumon de renard), faisant allusion au mammifère carnivore bien connu des basses-cours, dont la valeur respiratoire en course à pied n'est plus à démontrer.

\section{Les plantes : Éphédra, Coca, Khât, Iboga, Maté...}

Les athlètes avaient également recours aux plantes. Chez les Grecs, qui attribuaient l'apparition du point de côté à ... la dilatation de la rate, les coureurs absorbaient, rapporte Pline l'Ancien, une décoction savante à base de prêle, une plante très commune appartenant à la famille des équisetacées. Ils devaient d'abord s'abstenir de consommer toute matière grasse pendant vingtquatre heures, puis boire trois jours durant par doses régulières la décoction préparée dans un récipient de terre n'ayant jamais servi. Ce breuvage, fait par réduction aux deux tiers, avait, croyaient-ils, le pouvoir de consumer l'encombrante rate!

Plus brûlant était le procédé recommandé par Hippocrate qui prescrivait d'appliquer dans la zone de la rate des champignons séchés, et de les enflammer... Beaucoup plus tard, au XVI siècle, on alla encore plus loin et cette fois sans utiliser ni herbes, ni cryptogames, en procédant à une expérience d'ablation pure et simple de la rate sur des chiens. Tous en moururent, ce qui dissuada provisoirement les hommes d'essayer à leur tour et leur valut une sévère critique dans le sérieux Journal de Trévoux. Cependant, un siècle après cette tentative, le médecin allemand Godefroy Moebius [1] fit état de pratiques analogues visant à la suppression de la rate sur des coureurs de la ville d'Haberstadt. On leur brûlait la rate à vif et, dit-on, sur trois opérés, deux survécurent pour "courir comme des dératés". La suppression de cet organe hématopoiétique, qui dans l'ancienne médecine passait pour sécréter "la bile noire", doit être assimilée à un placebo à ranger aux côtés de ceux, tout aussi folkloriques, sévissant aux premiers jours du $20^{\mathrm{c}}$ siècle.

A cette époque, on estimait que les bonnes perfor- mances d'un marcheur étaient obligatoirement dues à la possession de la "Jarretière du diable" (1) dans la composition de laquelle entraient entre autres de la peau de loup et des cheveux de pendu, ou de la "ceinture magique", ou du "bâton du voyageur" qui rendait infatigable celui qui le portait et le préservait de l'attaque des brigands, des chiens enragés, des bêtes féroces et... de la rapacité des hôteliers !

Revenons aux plantes : leur utilisation est très ancienne aussi bien en médecine qu'à des fins plus ambiguës dans les filtres, mortels, aphrodisiaques ou stimulants. De nombreux peuples primitifs s'en servaient. Et, comme l'a constaté Lahy-Hollebecque :

"À tous les étages de la civilisation, l'honme organise ses paradis artificiels."

Selon la mythologie scandinave, les Berserks absorbaient une drogue nommée Butotens, extraite de l'amanite tue-mouche (Amanita muscaria). Ce champignon très commun contient en effet de la muscarine et de la muscaridine, substances qui provoquent entre autres des troubles nerveux, des accès de colère, une ivresse apparente pouvant aller jusqu' au délire, le tout accompagné d'hallucinations. Pour les Berserks, le Butotens permettait d" "augmenter leur puissance douze fois"

La médecine chinoise fait, elle aussi, grand cas des plantes depuis des millénaires. La racine de ginseng, dont la forme évoque curieusement un corps humain, est utilisée pour ses vertus puissamment toniques comme une panacée fortifiante et défatigante. Pour accroître la résistance cardiaque et la pression sanguine, les Chinois se servaient également des substances extraites du "ma huang", un arbrisseau qui est notre éphédra. Ils avaient, ce faisant, découvert l'éphédrine qui ne fut chimiquement identifiée qu'en 1924

Une peinture rupestre remontant à l'aube des temps historiques chinois, il y a quelque cinq mille ans, représente l'empereur du moment mâchant des feuilles d'éphédra pour y puiser la force d'accomplir sa tâche car, explique le Pr V. Demole (2) :

"L'empereur n'a pas le droit d'être fatigué. Il se doit entièrement à l'état, il reste constamment éveillé."

Depuis, l'usage de l'éphédrine, cet alcaloïde stimulant, s'est répandu aussi chez les sportifs.

Dans ce qui allait devenir 1'Amérique latine, Ies peuples indiens n'étaient pas en reste. Certains employaient les feuilles du coca, un arbuste du Pérou dont on tire la cocaïne, aux propriétés toniques et antinévralgique. Ils s'en servaient à des fins religieuses mais également pour entreprendre de longs parcours, au point d'en faire une unité de mesure, la cocada, distance parcourue sous l'effet d'une dose de coca!

Sur les hauts plateaux de l'Équateur, on connaissait 
aussi depuis longtemps les particularités d'une variété locale de gentiane surnommée Cashpa china rjugo, c'est-à-dire, "l'herbe qui fait courir". Les Incas l'avaient adoptée pour ne pas sentir la fatigue.

Au Mexique, les Indiens consomment encore des champignons hallucinogènes des genres Striopharia et Psilocybe, dont les effets sont également proches de ceux du peyotl, un cactus poussant en Amérique Centrale, qu'utilisaient les Indiens pour parcourir de grands trajets, à l'époque de la conquête menée par Cortès.

En Afrique, les feuilles de khât avaient la même destination pour les habitants des bords de la mer Rouge, tandis qu'au cœur du continent noir, certaines tribus puisaient dans la racine d'iboga la force nécessaire aussi bien aux longs parcours de chasse qu'aux interminables danses rituelles.

Le célèbre médecin missionnaire français Albert Schweitzer (3), exerçant à Lambaréné au Gabon pendant la première moitié $\mathrm{du} \mathrm{XX}^{\mathrm{e}}$ siècle, a bien décrit les effets roboratifs de ce végétal :

“... Les gens du pays peuvent, après avoir consommé certaines feuilles ou racines, pagayer vigoureusement tout un jour sans ressentir ni faim, ni soif, ni fatigue et en manifestant une gaieté et un entrain toujours croissants".

Il est remarquable que les Pahorins (peuple noir du Gabon) qui connaissent et récoltent la noix de kola, n'utilisent pas cette dernière qui est exportée et lui préfèrent la racine d'iboga.

On est déjà bien loin, avec ces pratiques, des vertus que les Grecs cherchaient dans les plantes mellifères à travers l'hydromel, et que les Romains trouvaient dans la sauge, plante miracle qui faisait dire aux sages de l'École de Salerne: "Cur moriatur homo, cui salvia crescit in horto ?" (Pourquoi l'homme meurt-il, quand la sauge pousse dans son jardin?).

\section{Breuvages magiques et potions accélératrices}

Sautons quelques siècles : comme il y a une ère moderne du sport, il y a une ère moderne du dopage, la coïncidence ne devant rien au hasard. La légende tenace de la rate a prouvé la permanence du désir d'améliorer les possibilités humaines à n'importe quel prix, mais plus on avance dans le temps et plus les recettes deviennent sophistiquées.

Il y eut bien, au début du XIX' ${ }^{c}$ siècle, le célèbre pedestrian anglais, le capitaine Robert Barclay Allardyce, pour préconiser gentiment de boire "de la bière forte" afin d'affronter les courses de longue haleine en vogue à l'époque dans les îles britanniques. Mais tout le monde n'écoutait pas ses conseils, à commencer par ses rivaux. En 1807, dans un match défi sur vingtquatre heures, son adversaire Abraham Wood reconnut avoir absorbé du laudanum, c'est-à-dire une teinture d'opium, ce qui ne l'empêcha pas d'abandonner...

Rapidement, ce sont les cyclistes qui ont brandi bien haut le triste flambeau du dopage. Il est vrai que la création et la multiplication des épreuves de six jours les y encouragea grandement. Les malheureux cherchaient d'ailleurs autant à lutter contre la fatigue qu'à améliorer leurs possibilités : au siècle dernier, ces premiers six jours ne se disputaient pas encore par équipes de deux coureurs chargés de se relayer, de même que le temps passé en poste sans repos n'avait pas fait l'objet d'une limitation.

Les Américains étaient friands de ces courses et des milliers de New-Yorkais se bousculaient pour les suivre, aux portes du tout nouveau Madison Square Garden. L'Amérique achevait bien les chevaux mais ne dédaignait pas voir les cyclistes aller au bout de leurs forces, augmentant au besoin la durée de leur calvaire. C'est ainsi qu'en 1896 la foule qui assistait aux premiers six jours du Garden exhorta le vainqueur, l'Irlandais Teddy Hale, à parcourir dix miles de plus après son arrivée. Épuisé, Hale s'exécuta - le mot n'est pas trop fort - jusqu'à être victime d'hallucinations. Peu de temps avant, un autre concurrent, Major Taylor, avait été, sous l'effet de la fatigue ou des "remontants" pris d'une véritable crise de folie et, jetant bas sa machine, avait attaqué des spectateurs...

Dans l'ombre de ces pauvres héros s'activaient déjà les "soigneurs", personnages louches qui n'ont jamais quitté l'arrière-plan de la scène cycliste et qui, sous des dehors quelque fois pseudo-scientifiques, sont de redoutables charlatans. Même de nos jours, des journalistes, des champions apparemment évolués n'hésitent pas à servir du "docteur" à certains de ces soigneurs, et plus encore, pour les seconds, à se fier aveuglément à leurs "ordonnances"...

La plume étincelante de Pierre Chany a ciselé un savoureux mais terrifiant portrait de ces soigneurs, dans sa Fabuleuse histoire du cyclisme (4) :

"Ces personnages souvent pittoresques, et douteux parfois, constituent le folklore de la course. Ils sont très conscients de leur importance. Ainsi, les soigneurs, dont Pierre Viel est le porte-drapeau, Joseph Torsin et son cigare, le vieux Giuseppe Leoni et son charabia franco-italien. Léon Sonnet, Guerlache, etc.

Dans sa jeunesse, Pierre Viel a fait des études en pharmacie, qualité qui lui confère une grande autorité auprès des coureurs! Il a du bagout, Pierre Viel. Sa force de persuasion est exceptionnelle, ses connaissances médicales suffisantes pour lui permettre de réveiller un "moribond" en moins de dix minutes! Les résultats sont parfois moins heureux. Un soir, 
Armand Blanchonnet tourne de l'ceil. Il faut le transporter d'urgence à l'hôpital Boucicaut où il sera sauvé de justesse : le soigneur a forcé la dose!

Le meilleur ami de Viel, son compère, c'est Fernand Trignol, qui s'intitule lui-même "Roi des truands". Il a pour fonction officielle de faire visiter le campenent aux personnalités désireuses d'approcher les champions - "Ici le domaine de la Brinvilliers!" dit-il en désignant la "guitoune" de Vielet pour occupation plus clandestine de "tondre les caves "à la passe anglaise ou au poker dans une cabine désaffectée du boulevard de Grenelle."

À l'époque héroïque, quand ils se contentaient de faire boire de la caféine à leurs poulains, le mal n'était pas si grand, mais ces alchimistes au petit pied n'avaient guère de scrupules avec leurs cobayes.

Le plus fameux d'entre eux, à la fin du siècle, fut I'Anglais Choppy Warburton, un ancien coureur à pied qui avait trouvé moins fatigant et plus rentable de faire courir les autres à vélo. Le Gallois Jimmy Michael, prince de la vitesse, ses compatriotes les frères Arthur et Tom Linton, furent les principales vedettes de l'écurie Warburton.

Le journaliste anglais C.H. Stancer (5) rapporte que les exploits de ces cracks étaient généralement attribués aux mystérieuses potions de Choppy Warburton.

"Les champions bénéficiant des soins de Choppy étaient sujets à des éclairs de forme absolument transcendants, suivis de défaillances insurnontables et dramatiques, ce qui correspond exactement aux symptômes d'un dopage administré par une personne n'ayant que des notions rudimentaires de médecine. Un des breuvages de Choppy était la "Cuca Cup", sorte de mixture chocolatée que l'on disait à base de cocaine..."

Le journal Paris Vélo décrit Choppy Warburton dans son numéro de magicien :

"De sa poche soudain, on le voit tirer une fiole minuscule, il la montre à son coureur, la débouche avec un luxe inoui de précautions, verse la mixture inconnue qu'elle contient dans une boîte à lait et, toujours courant, bousculant qui le gêne, s'en va à l'autre bout de la piste pour la passer à Linton, avec un sourire entendu. C'est de la drogue, disent les uns, c'est du bluff disent les autres: drogue ou bluff, c'est tout un..."

Cependant, un jour, au cours d'une épreuve de vitesse, Warburton utilisa son redoutable talent à rebours : comme il avait parié sur... l'adversaire de Jimmy Michael, il fit prendre à ce dernier une potion qui devait ressembler à un "bouillon de onze heures". Michael dut abandonner l'épreuve et Warburton y laissa sa réputation. Il fut même disqualifié pour le restant de ses jours. Ceux de Jimmy Michael, malheureusement, étaient comptés. Le Gallois devait mourir à vingt-huit ans d'une crise de delirium tremens, à bord du paquebot qui l'emportait vers une tournée américaine.

Que pouvait-il y avoir dans les fioles de Choppy Warburton? Nul ne 1'a su exactement, car il est mort avec son secret. Paul Hamelle (6), reprenant l'idée de placebo avancée dans Puris Vélo, avança lui aussi, dans Le Miroir des Sports, que Warburton n'avait peut-être aucun secret et se contentait de recourir à la seule magie de l'effet psychologique :

"Je me suis laissé dire (écrivait-il) que le mystérieux breuvage n'était que de l'eau claire agissant sur le buveur non par voie de digestion mais de suggestion. Le certain est que l'effet moral sur les spectateurs - je n'ose dire les compétiteurs - était considérable : ils regardaient hypnotisés."

Faute de preuve, on ne peut établir de relation entre l'éventuelle eau claire de Choppy Warburton et le delirium de Jimmy Michael. Et C.H. Stancer (5) conclut prudemment :

"Arthur Linton et Jimmy Michael sont morts jeunes, mais je ne me permettrai pas de dire que l'action de Choppy Warburton y fut pour quelque chose."

\section{Les topettes du $\mathrm{XX}^{\mathrm{e}}$ siècle : Caféine, Strychnine, Cocaïne, Arsenic, ...}

C'est au cours de ces années charnières entre XIX" et $\mathrm{XX}^{\mathrm{e}}$ siècles que les fioles de Choppy Warburton et de ses collègues prirent le nom de "topettes". Venue du langage picard, avec la même racine que toupie, la topette désignait une petite bouteille longue et étroite, ainsi que, par extension, son contenu.

De nos jours, la topette a encore cours dans le jargon cycliste. Elle a pris la forme d'un flacon plat qui se glisse facilement dans la poche dorsale d'un maillot cycliste. On en trouve en vente dans la plupart des boutiques de cycles.

Au temps de Warburton, et malgré le mystère dont il entourait sa propre méthode, on sait pour l'essentiel ce qui constituait les cocktails des coureurs, leurs "potions magiques", tel cet american coffee qui donnait des ailes aux "écureuils" du Garden de New York et d'ailleurs.

Avec la caféine, la strychnine et la cocaïne, les poètes du vélo trouvaient des rimes riches à souhait! Certains sorciers ajoutaient à cet arsenal de l'arsenic : en médecine, en effet, on utilisait comme tonique contre le lymphatisme la liqueur de Fowler - de l'arsénite de potasse - à très petite dose. Inconscients du danger, les cyclistes en buvaient à pleines gorgées. De quoi réveiller un mort, après l'avoir tué ! Dans le même style, la liqueur de Pearson - arséniate de soude - avait également ses amateurs.

On note avec intérêt qu'au début du siècle le recours à ces produits n'avait rien de clandestin, au contraire. Choppy Warburton en faisait, on l'a vu, un numéro à grand spectacle et la publicité sur ces mixtures fleuris- 
sait partout. La "Kola-Bah-Natton", expérimentée avec succès par l'Académie de Médecine en 1890, était proposée comme tonique régulateur du cœur, comme un puissant régénérateur "quintuplant la force de résistance à la fatigue et supprimant l'essoufflement."

D'autres produits avaient, selon leur créateur, des propriétés spécifiques sur une qualité physique : Harry Leeming, entraîneur de grande renommée pendant les années 1890 , avait mis au point un "élixir de vitesse". De même, une annonce de 1892 proposait la potion Coca des Incas "pour un démarrage foudroyant".

Dans la revue londonienne Abbotempo, le spécialiste américain de la drogue et du dopage Max M. Novich (7) a décrit différents produits, plus ou moins stimulants mais sûrement pas inoffensifs, en vogue autrefois :

"La plupart des coureurs des six jours étaient de grands buveurs de café noir. Certains avaient toujours à portée de main une bouteille de café corsé par quelques gouttes de Peppermint ou par des pastilles de caféine pour lutter contre la somnolence et la fatigue. D'autres coureurs préféraient le thé fort, arrosé de brandy (eau-de-vie). Les entraineurs dosaient le café et le thé selon les efforts que leur coureur allait devoir fournir et il était notoire que les deux derniers jours de l'épreuve, une forte dose de brandy était ajoutée au café ou au thé. Pour les sprints, les coureurs prenaient des capsules de nitroglycérine afin de diminuer la durée et l'intensité de la dyspnée, fréquente après un effort violent. Souvent, ils ne craignaient pas d'utiliser la cocaïne. Mais son efficacité dépendait du dosage et de la façon dont elle était administrée : généralement par voie buccale, mélangée à de la caféine. Les entraîneurs de la vieille école qui fournissaient ces mixtures à base de cocaïne déclaraient avec assurance qu'un coureur fatigué par une course de six jours retrouvait son deuxième souffle après l'absorption de ces mixtures."

Certains produits étaient préparés et vendus spécifiquement pour les cyclistes. En 1892, le breuvage au nom évocateur de "Vélo-Guignolet" faisait fureur. Composé d'après la recette attribuée à Jeanne de la Noue, fondatrice, en 1704, de l'ordre des sœurs de la Providence de N.-D. des Ardilliers, c'était, selon la réclame de l'époque, "le breuvage le meilleur, le plus tonique, le plus efficace". Ses précieuses qualités l'avaient fait adopter par les velocemen de France.

'La nitroglycérine est un composé chimique tiré de la glycérine. C'est un liquide huileux, jaunâtre, doué de propriétés explosives au plus léger choc. Ce nitrate organique est utilisé en thérapeutique dans le traitement de l'angine de poitrine (obturation temporaire des artères coronaires). On l'emploie sous sa forme trinitrée - c'est la trinitrine - qui a un effet vasodilatateur. C'est un rôle analogue que l'on attribue, avec un peu trop de complaisance, au whisky. Pour les coureurs, la nitroglycérine était censée diminuer les difficultćs respiratoires et l'essoufflement à l'effort - ce que l'on nomme dyspnée.
En 1894, le docteur Léon Petit (membre du conseil d'administration du Touring Club de France) tenait une consultation médicale dans l'hebdomadaire sportif La Bicyclette $(8,9)$. À la question d'un lecteur l'interrogeant sur les produits facilitant l'effort de longue durée, il avait répondu :

"Adressez-vous à $M$. Grenet, pharmacien, 20 rue des Sablons à Paris, et demandez-lui de vous préparer la potion. stimulante qu'il a fournie à Louis Cottereau lors de la dernière course Bordeaux-Paris ${ }^{2}$.Vous en prenez un verre à liqueur toutes les quatre heures pendant la course".

La potion stimulante préconisée par le Dr Petit portait le nom évocateur d'élixir Saintonger (10). Ce breuvage préparé avec du maté - une boisson stimulante obtenue par torréfaction et infusion de "thé du Paraguay", variété de houx sud-américaine - agissait avec précision sur l'appareil respiratoire. Selon son fabricant, elle était parfaite pour "donner du souffle".

L'effort surhumain demandé aux coureurs cyclistes et les solutions qu'ils tentaient de trouver eux-mêmes ou avec l'aide de leur entourage devaient trouver un écho très spectaculaire dans le Tour de France en 1924, lors de la rencontre entre un grand reporter, Albert Londres (11), et deux grands champions, les frères Henri et Francis Pélissier.

\section{Les Forçats de la Route}

$\mathrm{Au}$ départ de la troisième étape du Tour, à Cherbourg, Henri, qui a gagné la précédente édition de l'épreuve, est importuné par un commissaire de course tatillon lui reprochant de porter deux maillots superposés au départ et d'en retirer un en cours de route, ce que le règlement interdit. Ulcéré, Henri ordonne un peu plus loin à son frère de quitter la course sur-le-champ. Albert Londres, cherchant les Pélissier sur le parcours, apprend leur abandon et les retrouve au Café de la Gare, à Coutances.

Les deux Pélissier sont là, en effet, et exhalent leur rancœur, que le journaliste traduit le soir même dans un papier à sensation titré "Les Forçats de la Route". Henri y raconte l'incident du départ à Cherbourg, puis il enchaîne devant son auditeur ravi :

"Vous n'avez pas idée de ce qu'est le Tour de France. C'est un calvaire. Et encore, le chemin de croix n'avait que quatorze stations tandis que le nôtre en compte quinze. Nous souffrons sur la route, mais voulez-vous savoir comment nous marchons? Tenez..."

De son sac il sort une fiole: ça, c'est de la cocaïne pour les yeux, et ça du chloroforme pour les gencives. Et des pilules, voulez-vous des pilules? Les frères en sortent trois boîtes chacun.

Bref, dit Francis, nous marchons à la dynamite!"

\footnotetext{
${ }^{2}$ Louis Cottereau, un sprinter (!) bourguignon, avait gagné l'épreuve en 1893 ; mais en 1895 , c'est un Danois marchand de cycles à Dieppe, Charles Meyer, qui l'emporta.
} 
Ce faisant, les rusés duettistes avaient aussi profité de l'oreille attentive mais inexperte d'Albert Londres pour laisser filtrer toute leur mauvaise humeur à l'égard de l'organisateur du Tour, Henri Desgrange. Pierre Chany (4) estime que le journaliste, humaniste mais étranger au phénomène du sport, s'est laissé prendre au jeu.

"Londres était un fameux reporter mais il ne savait pas grand-chose du cyclisme", dira beaucoup plus tard Francis Pélissier, devenu directeur sportif de l'équipe La Perle. "Nous l'avons un peu bluffé avec notre cocaine et nos pilules! Ça nous amusait d'emmerder Desgrange. Cela dit, le Tour de France, en 1924, c'était pas de la tarte!"

On croit volontiers Francis pour ce qui est de chatouiller Henri Desgrange et pour la tarte. Mais la suite des évènements incline à plus de réserves sur la véracité des fausses confidences à base de pilules.

Le dopage, en tout cas, ne va plus quitter le devant de la scène, qu'il s'agisse de cyclisme ou d'autres sports gagnés petit à petit par la gangrène, à coup de scandales vérifiés ou de rumeurs insidieuses... car le mal est tel qu'il finira par entraîner la suspicion dès qu'un exploit sortira de l'ordinaire ou dérangera l'ordre établi. Avec l'avènement du sport comme phénomène social du $X X X^{c}$ siècle, le dopage devient aussi bien un moyen de triompher qu'une excuse à ses propres échecs. Dans ce dernier cas, le dopé, c'est évidemment l'autre...

\section{La contribution olympique}

Les Jeux olympiques de l'ère moderne, restaurés par le baron Pierre de Coubertin en 1896, ne tardent pas à verser leur contribution à notre histoire. En 1904, à SaintLouis, aux Etats-Unis, le marathon se dispute sur un parcours écrasé par la chaleur et la poussière...

L'Américain Fred Lorz figure en bonne place dans le groupe de tête lorsque des crampes le terrassent. Il abandonne, monte à bord d'une voiture qui file vers l'arrivée. Mais à huit kilomètres du but, Lorz se sent mieux et, sans complexes, descend de voiture pour terminer le parcours. Sur le stade, il évite de détromper ceux qui l'acclament et, jusqu'à l'arrivée du vrai vainqueur, l'Américain Thomas Hicks, il fait illusion!

Un officiel rétablit alors la vérité, accusant Lorz de tricherie. Hicks a donc gagné. Il est champion olympique, mais non sans peine et sans une toute autre forme d'assistance... Son entraîneur, Charles Lucas (12) raconte en effet qu'à quelques kilomètres de l'arrivée, son coureur a, lui aussi, été victime d'une soudaine défaillance.

"Je décidais alors de lui injecter un milligramme de sulfate de strychnine et de lui faire boire une large rasade de brandy. Il repartit tant bien que mal et il fallut avoir recours à une seconde injection à quatre nilles du but pour que Hicks reprenne un semblant de rythme de course et termine son parcours..."
Il faut croire qu'à l'époque la menace du dopage sur la santé des athlètes comme sur l'équité sportive n'a pas encore pénétré les mœurs car, après ce marathon mouvementé, le rapport officiel de la course juge bon de voir dans ces faits un côté "positif" qui n'a pas le sens donné de nos jours au résultat d'un contrôle ! :

"Le marathon (dit ce communiqué) a démontré du point de vue médical que les drogues peuvent être très utiles aux athlètes en cours d'épreuve."

Coubertin qui est resté en Europe et dénonce les nombreux scandales de Saint Louis, ne se leurre pas sur le danger qui monte :

"Deux aspects de ces Jeux doivent nous donner à réfléchir (écrit alors le baron) : le trucage et la mascarade. Une performance est truquée quand elle est le fruit d'un entrainement devenu l'alpha et l'omega d'une existence, et quand on dope l'athlète comme un cheval..."

L'anathème du baron ne s'adresse évidemment pas à Lorz, mi-tricheur, mi-farceur, dans le style du plaisantin qui devança Frank Shorter en 1972 sur la ligne d'arrivée du marathon des Jeux de Munich, mais à Hicks, le vainqueur...

Aux Jeux de 1932, à Los Angeles, on joue sur un tout autre registre et sur un tout autre terrain. En natation, la razzia des Japonais a un avant-goût de Pearl Harbour sur des eaux jusqu'alors largement contrôlées par la flotte des nageurs américains. Dans le bassin californien, les Nippons gagnent le 100 mètres avec Yasuji Miyazaki, le 1500 mètres avec Kusuo Kitamura, qui n'a que quatorze ans et demi et dont le record "vivra" plus de 20 ans, le 100 mètres dos avec Masaji Kiyokawa, le 200 mètres brasse avec Yoshiyuki Tsuruta et enfin le relais 4 × 200 mètres, qui laisse l'équipe américaine à plus de douze secondes !

Sur les six épreuves masculines du programme de natation, l'équipe du Japon rafle cinq titres olympiques, abandonnant seulement le 400 mètres à Clarence "Buster" Crabbe. Dans le camp américain, on digère mal l'humiliation, et tout ce qui peut l'expliquer est bienvenu ! On attribue volontiers le raz de marée japonais à l'emploi d'une technique nouvelle - l'oxygénothérapie - qui consiste à faire inhaler de l'oxygène à un nageur avant le départ de l'épreuve.

On parle aussi de mystérieuses fioles découvertes dans les vestiaires des Japonais après les épreuves : l'analyse de ces fioles aurait révélé la présence, entre autres produits chimiques, de trinitrine... Naturellement, on ne manque pas de faire remarquer que l'entraîneur japonais Matsuwada est, dans le civil, professeur de chimie à l'université de Tokyo...

On ne prête qu'aux riches! Ce faisant, on oublie aussi de dire que la natation japonaise doit sa réussite à l'effort qui a été fait pour ce sport dans tout le pays, au point d'en avoir fait un sport national. On passe sous silence le fait que Matsuwada est un entraîneur de qualité doublé d'un observateur remarquable. 
Il a, selon ses propres critères, modifié le battement de jambes de ses nageurs pour le rendre efficace dans les deux sens. Mais comme on le constatera souvent par la suite, les battus auront de plus en plus tendance à oublier les éléments objectifs de leur défaite pour s'abriter - à tort ou à raison - derrière le dopage de l'adversaire.

La technique de la rumeur a encore fait ses preuves et toujours en natation, lors des Jeux olympiques de Montréal où les nageuses américaines furent sévèrement dominées par celles d'Allemagne de l'Est. Curieusement, deux ans plus tard, lors des championnats du monde organisés en 1978 à Berlin, les Américaines triomphantes ne firent pas la moindre allusion au dopage éventuel de leurs rivales. Il est vrai que dans ces championnats, les nageurs soviétiques avaient, contrôle positif à l'appui, pris le relais !

Entre-temps, de Los Angeles (1932) à Mexico (1968), le dopage avait changé d'ère, passant insensiblement du stade artisanal et empirique à celui de l'industrie pharmaco-scientifique détournée de son cours.

Alors que la partie purement historique s' achevait, la chimiothérapie le faisait entrer dans l'histoire contemporaine.

\section{L'avoine enchantée envahit les hippo- dromes}

Mais pour être complet sur cette "vieille histoire", on ne peut manquer de souligner les routes parallèles du dopage des sportifs et du dopage des chevaux. En effet, dans le même temps où l'homme s'efforçait d'améliorer ses propres possibilités physiques en empruntant des chemins détournés, il suivait pour sa plus noble conquête des voies elles aussi cavalières.

Ceux qui ont vu sur grand écran la formidable course de chars de Ben Hur comprendront mieux la fureur dégagée par ces formules 1 antiques dont les chevaux étaient abreuvés d'hydromel : en place pour le quadrige ! Avec le temps, l'imagination des propriétaires et des cavaliers devait montrer ses possibilités. Dans son traité De l'Agriculture, écrit au XVI' siècle, Varron rapporte que les hippiatres - c'est-àdire les vétérinaires spécialisés dans les maladies du cheval - recommandaient, pour donner du punch à leurs patients, de leur faire absorber du miel, de la semence d'anis, et même de la sandaraque, une résine tirée du thuya...

Quelquefois, les effets des potions dépassaient largement les prévisions du préparateur. Dans ce cas, le cheval n'était pas seul à faire les frais de l'opération. En Angleterre où, en apparence du moins, on ne badine pas avec la santé des cheveux, on pendit haut et court Daniel Dawson, coupable d'avoir forcé la dose et expédié quelques chevaux de race au paradis des pur-sang ! C'était en $1810 \ldots$ À cette époque, le folklore avait encore sa place dans le dopage équestre. Dans la tradition du miel fermenté des Romains, certains n'hésitaient pas à faire partager à leurs chevaux le goût des boissons alcoolisées ou simplement stimulantes : thê, café, vin blanc, cidre, cherry, whisky, champagne.

Ces pratiques n'étaient pas seulement destinées à des triomphes en course. La revue hebdomadaire omnisports Sport universel illustré (13) donne, en 1903, l'exemple de contrebandiers qui opéraient sur la frontière belge et faisaient boire à leurs montures "une infusion très forte de café ou de the fortement alcoolisê" pour mieux échapper aux poursuites douanières!

Au XIX ${ }^{e}$ siècle, les entraîneurs américains dont la corporation florissante était liée au développement du turf, étaient à 1'affût de tous les procédés capables d'embellir leur carte de visite. C'est ainsi que les alcaloïdes, déjà utilisés spontanément par certaines tribus indiennes d'Amérique latine, furent enrôlés dans l'aventure.

Vers 1890 , l'usage de la cocaïne, de la morphine, de l'atropine et de l'héroïne était devenu courant. Il devait se répandre lentement à travers les pays de tradition équestre : Angleterre en 1836, Russie, Autriche en 1899, Allemagne et France en 1900. Dans ces pays, les succès des chevaux américains devaient être en grande partie attribués au dopage. On oubliait comme on allait le faire plus tard à propos des nageurs japonais, de prendre en compte également la qualité intrinsèque des chevaux, celle de leur ferrure, et la rigueur des nouvelles méthodes d'entraînement développées par les entraîneurs d'outre-Atlantique.

Le dopage, cependant, était une réalité, assortie d'un certain nombre de gadgets comme l'emploi de produits vésicants appliqués sur les cuisses, ou la mise en place d'appareils fixés au sulky et envoyant des décharges électriques au cheval. Au début du XXe siècle, les chroniques abondent en récits dramatiques sur les accidents et les morts subites de chevaux dopés, dans les courses de vitesse comme dans les raids d'endurance. Dans le Journal des vétérinaires britanniques, Robert G. Mars (14) consigne ses expériences personnelles et constate que dans les années 30, aux Etats-Unis, les écuries de course ressemblaient à des pharmacies où l'on usait couramment de tous les alcaloïdes en vogue à l'époque.

D'autres études vétérinaires sur le dopage des chevaux attestent du pouvoir détonant des préparations utilisées. Certaines sont connues, comme les pilules contenant à la fois caféine, hérö̈ne, acide salicylique et conchonine (un alcaloïde extrait des quinquinas). Certaines étaient tenues secrètes, â la manière des potions de Choppy Warburton. Telle était la "Merveille médicale du Dr Wonder" ! Comme pour les sportifs, la Seconde Guerre mondiale devait amener le monde du turf aux ressources infinies de la chimiothérapie. Par chevaux interposês, les hommes n'en finissent décidément pas de vouloir gagner des courses... et des paris ! 


\section{La guerre de 30 ans : 1968- 1998}

Les Jeux de Grenoble et Mexico en 1968 furent les premiers à instituer un contrôle antidopage des athlètes. Ce fut le début d'une longue guerre entre les voleurs de podiums et les gardiens de l'éthique où chaque bataille porte le nom d'une molécule. Pendant ces trente dernières années, l'histoire contemporaine du dopage va se décliner au rythme des performances des toxicologues qu'ils soient dans le camp des tricheurs ou dans celui de la lutte antidopage.

\section{Une bombe à retardement}

Le dopage a complètement envahi la sphère sportive au point de poser la question de sa survie. Certes, il est difficile de se faire une photographie exacte de la situation. On ne saura la vérité que dans 10 ou 15 ans lorsque enfin les langues se délieront. Mais les témoignages qu'on recueille à présent sur le sport des trois décennies passées laissent présager le pire. On pense évidemment au rapport du juge Charles Dubin ${ }^{3}$ ou au livre de Charlie Francis (15) qui firent suite à l'affaire Ben Johnson. Mais il y a aussi le témoignage de Brigitte Berendonk (16), ex-athlète de RDA. Ou l'exhumation des dossiers secrets de la Stasi. De même, l'affaire Festina du Tour de France 1998 ne laisse planer aucun doute sur l'importance du fléau. Tout cela tranche avec les propos rassurants du pouvoir sportif qui invoque le très faible pourcentage d'athlètes positifs. C'est exact. Sur les 16872 tests effectués depuis les JO d'hiver de Grenoble, 53 seulement ont donné lieu à des sanctions, soit 0,31 pour cent ! Mais il ne faut pas se leurrer. Ce chiffre est ridiculement bas par rapport à la réalité du dopage. Les experts de la chose athlétique, champions et médecins, ne s'y trompent pas. Selon Pat Connolly (17), ex-pentathlète olympique et entraîneur de l'équipe féminine américaine aux JO 1984: "Le dopage concerne 30 pour cent des athlètes féminines américaines". L'Anglais David Jenkins (18), finaliste au $400 \mathrm{~m}$ en 1972, précise: "Un tiers des athlètes d'accord mais $63 \%$ des médailles!". Dans certaines disciplines, la situation est pire encore. Le Docteur Robert Kerr (19) estime ainsi que "90\% des sprinters de l'élite internationale sont dopés". Ce décalage entre les chiffres officiels et la réalité du terrain s'explique très

\footnotetext{
${ }^{3}$ Responsable de la "Commission d'enquête sur le recours aux drogues et aux pratiques interdites pour améliorer la performance athlétique" qui fut mise en place par le gouvernement canadien après le scandale provoqué par la disqualification de Ben Johnson aux Jeux de Séoul en 1988. (17)
}

bien lorsqu'on sait que plusieurs substances dopantes sont toujours indétectables : hormone de croissance, corticoïdes naturels, érythropoḯtine, ACTH, etc. Et si des progrès se confirment dans les méthodes de détection de certaines de ces substances-là, on peut imaginer que demain, d'autres apparaîtront sur le marché, probablement des précurseurs ou des dérivés de ces mêmes molécules qui obligeront de tout reprendre à zéro. Voilà l'enseignement principal que l'on peut tirer du survol de trente années de lutte.

\section{0 : les années amphétamines}

En 1968, le Comité International Olympique institue les contrôles antidopage pour répondre à une situation inquiétante. Les athlètes puisaient un peu trop facilement dans les armoires à pharmacie. Remarquons qu'au début, les tests avaient plutôt un but dissuasif. On ne détectait que certaines amphétamines (Maxiton', Tonédron ${ }^{\oplus}$ ) et encore lorsqu'elles étaient consommées à des doses de cheval. En outre, différentes techniques avaient vu le jour pour tromper la surveillance très laxiste des contrôleurs : mélange d'additifs à l'urine, siphonnage de la vessie, système de pipette cachée, etc. Dans un tel contexte, on ne s'étonnera pas que les contrôles à Grenoble (86 tests) et à Mexico (668 tests) ne pincèrent qu'un malheureux pentathlète suédois... parce qu'il avait trop bu ${ }^{4}$. Pas de soucis, en revanche, pour ceux qui carburaient aux stérö̈des anabolisants, aux corticoïdes ainsi qu'aux amphétamines et apparentées (pémoline, pipéridine). Ces dernières surtout posaient problème. N'oublions pas qu'un an plus tôt, le cycliste Tom Simpson avait trouvé la mort sur les pentes du Mont-Ventoux. Comme d'autres avant Jui, l'Anglais avait été victime du triptyque: effort intense, zhaleur, amphétamines. Mais ce décès en images eut l'effet d'un électrochoc. Il faut dire qu'à l'époque, les amphétamines n'étaient pas connotées aussi péjorativement qu'aujourd'hui. On les voyait, au contraire, parées de toutes les vertus, notamment en Angleterre, celle d'avoir gagné la guerre. Lorsque le 12 octobre 1940, Hitler abandonne son "Opération Otarie" pour la conquête du Royaume-Uni, un grand journal britannique avait titré: "La Méthédrine $e^{\oplus}$ a gagné ta bataille de Londres". Pendant cette période, le Ministère de l'Approvisionnement anglais avait distribué 72 millions de comprimés de métamphétamine, qui permirent aux Britanniques de supporter trois mois de bombardements intensifs et aux aviateurs de livrer une résistance

\footnotetext{
+ L'alcool est considéré comme dopage dans l'épreuve de tir car il permet de supprimcr les tremblements. L'équipe suédoise fut disqualifiée de sa troisième place qui revint à la France.
} 
héroïque. Ce médicament augmente en effet la confiance en soi, la volonté, la vigilance, etc. Dès les années 40 , on s'était aussi aperçu de son efficacité dans le cadre d'une performance sportive. Le 7 novembre 1942, le cycliste italien Fausto Coppi bat le record de l'heure du Français Maurice Archambaud grâce à "sept cachets d'amphétamine". (20) En 1950, le Maxiton ${ }^{\oplus 5}$ fait aussi partie de la pharmacie de la célèbre expédition "Annapurna Premier 8000" dirigée par Maurice Herzog (21.22.23). Comme toujours, il fallut attendre quelques années pour appréhender ses effets parfois désastreux. Les Jeux olympiques de Rome en 1960 avaient été endeuillés par la disparition du cycliste danois Knud Enemark-Jensen qui mourut d'avoir consommé des amphétamines et de l'acide nicotinique (vasodilatateur). En 1965, deux pays européens adoptèrent leurs premières lois antidopage : la Belgique, la France ${ }^{6}$, l'Italie, etc. Ce n'est pas un hasard si ces pays sont au premier rang des grandes nations cyclistes ! Le problème du dopage se pose là avec particulièrement d'acuité. En 1966, les premiers contrôles effectués en France révèlent des chiffres effarants: près d'un tiers des coureurs sont "positifs". Le mal est plus profond encore qu'on ne l'imaginait. On décide d'élargir le cadre de la lutte, ce qui entrâne une grève des coureurs du Tour de France. Mais rien n'y fait et les premiers cas de dopage aux amphétamines sont sanctionnés parmi lesquels le Belge Hermann Van Springel ou le champion de France de l'année 1967, Désiré Letort. Voilà dans quel contexte se situe l'année olympique 1968. Nous l'avons dit: les méthodes approximatives de détection ne permettent pas au début d'espérer de belles moissons de tricheurs. Les progrès viendront cependant assez vite. En 1974, treize cyclistes de premier plan (dont Walter Godefroot) sont déclarés positifs à une famille de substances jusqu'alors indécelables et proches parentes des amphétamines : les pipéridines (Lidépran $^{\circledast}$, Mératran $^{\circledast}$, Ritaline ${ }^{\circledR}$, etc.) Puis, trois ans plus tard, le Professeur Michel Debackere met au point une technique d'identification de la pémoline, une substance apparentée aux amphétamines, stimulant le système nerveux central, interdite mais jusque-là indécelable. Six gros bras, dont Eddy Merckx, Freddy Maertens et Michel Pollentier, se font épingler. Cela

\footnotetext{
5 Le Maxiton est la plus connue des amphétamines françaises. Il est apparu pour la première fois au Vidal (dictionnaire officiel des médicaments français) en 1949. Un an plus tard, on commercialisera la première métamphétamine française : le Tonédron ${ }^{(}$.

${ }^{6}$ Ironie du sort, il s'agit de la loi Herzog du nom du Ministre des Sports de l'époque, ex-alpiniste... et ancien consommateur de dopants ! [21]
}

marque la fin d'une époque. Certes, les amphétamines continuent de circuler dans les poches de sportifs. Dans une interview au Washington Post le 10 janvier 1990, l'Américain Carl Lewis affirme d'ailleurs :

"Qu'il ne fait aucun doute que Ben Johnson a pris des amphétamines aux Jeux olympiques de Séoul en 1988, pour pouvoir jaillir de la sorte lors de la finale du 100 mètres".

Mais il s'agit d'un usage risqué, dont l'élite se détourne progressivement, du moins en période de compétition.

\section{0 : les années anabolisantes}

L'histoire des anabolisants dans le sport commence évidemment avant le début des années 70. Rappelons que la mise au point du Dianabol ${ }^{\circledast}$ (métandiénone), premier stéroïde anabolisant utilisé par les sportifs, date de 1956. Déjà aux Jeux de Tokyo, en 1964, le recours aux stéroïdes est dénoncé aux dirigeants du CIO, parmi lesquels le jeune prince Alexandre de Mérode, qui vient tout juste d'être coopté, à trente ans, et qui soumet un projet d'étude au président de l'époque, Avery Brundage. Celui-ci est accepté et le prince de Mérode entre à la commission médicale jusqu'alors en sommeil. Il travaille à sa refonte qui sera terminée en 1967 et occupe sa présidence encore aujourd'hui. Car le lièvre levé à Tokyo n'est pas mince. On en aura la confirmation plus tard, lorsque le lanceur de marteau Harold Connolly (24), champion olympique aux Jeux précédents en 1956 à Melbourne, déclarera devant une commission d'enquête du Sénat américain en 1973 :

“Juste avant les Jeux de Tokyo, il semblait qu'autour de moi de plus en plus d'athlètes absorbaient des stéroïdes pour se préparer aux compétitions et l'ont avait l'impression de se placer dans une position nettement désavantageuse en ne s'embarquant pas dans le train de la médecine sportive..."

Dès lors, on décide d'interdire le produit. Cette décision est prise par la Fédération Internationale d'Athlétisme (IAAF) aux Jeux de Munich en 1972. Parallèlement, on s'efforce de trouver les moyens de déceler son usage. Des travaux sont conduits par le professeur britannique Arnold Beckett, membre de la commission médicale du CIO et cela débouche sur la mise au point du système de détection par son compatriote Raymond Brooks en 1973. À Montréal en 1976, les premiers tests sont effectués. On découvre 9 cas positifs au Dianabol ${ }^{\oplus}$. Pas mal. Pourtant ces résultats sont nettement sous-évalués dans la mesure où un athlète arrêtant sa cure 15 jours avant les compétitions ne pouvait être épinglé par les machines du CIO. En outre, seulement $15 \%$ des échantillons avaient été soumis à des tests de dépistage spécifiques aux stéroïdes anabolisants. Cela suffit néanmoins pour prendre conscience de l'ampleur du problème et, progressivement, des règlements anti-stéroïdes furent élargis aux autres disciplines, suscitant un nombre 
incalculable d'affaires à rebondissements. Aujourd'hui encore, la question de sécrétion endogène de la nandrolone divise le landerneau des spécialistes. Notons tout de même deux avancées significatives dans cette guerre aux anabolisants. D'abord, en 1987, une disposition réglementaire de la Fédération Internationale d'Athlétisme (IAAF) oblige les organismes membres à effectuer des tests inopinés, c'est-à-dire hors compétition, pour épingler les athlètes en phase de préparation. Ensuite, en 1991, on parvient à remonter plus loin dans le temps à la recherche du produit. Les stéroïdes anabolisants sont solubles dans la graisse. Le produit forme un dépôt dans les tissus, surtout s'il est administré par injection, qui peut être décelable vingt semaines durant, voire jusqu'a six a huit mois après la dernière prise. Enfin, un dernier paramètre complique la vie des tricheurs. Depuis 1997, plusieurs laboratoires ont pris la décision de retirer ces substances du marché, que ce soit en médecine humaine ou vétérinaire ${ }^{7}$. Des raisons commerciales mais également de détournement d'utilisation ont influencé cette décision.

\section{0 : les années testostérone}

Les risques liés à l'usage de substances synthétiques (stéroïdes anabolisants) ont guidé les athlètes vers des substances hormonales naturelles, dotées des mêmes effets. Les autorités s'en sont rapidement rendu compte et, en 1982, il a été décidé d'interdire aussi la testostérone. Seulement, les laboratoires se voyaient confrontés là à un problème quasiment insoluble. En effet, comment identifier l'apport frauduleux d'un produit qui se trouve sécrété naturellement par l'organisme ? Pour la première fois depuis sa création en 1966, les procédures d'analyses utilisèrent une notion quantitative. Les experts établirent un seuil critique au-delà duquel la suspicion de dopage paraît suffisamment forte pour donner lieu à des sanctions. On se fonde pour cela sur le rapport existant entre la testostérone et l'épitestostérone. Normalement, le rapport urinaire de T/épiT est voisin de 1. La testostérone exogène (frauduleuse) n'étant pas convertie dans le corps en épitestostérone, toute absorption médicamenteuse de l'hormone mâle augmentera le rapport. Un échantillon sera considéré comme positif si le rapport de la concentration totale de la testostérone à celle de l'épitestostérone dans l'urine dépasse 6. Pourquoi ce taux ? Il a été choisi suffisamment haut pour de ne pas sanctionner trop de faux positifs. En effet, les études ont montré que, sans dopage, $90 \%$ de la population avait un rapport de T/épiT $=1$ et pour $99,9 \%$ des individus, ce ratio était inférieur à six. Néanmoins, $0,1 \%$ (1 pour 1000 ) et même, selon d'autres études $0,8 \%$ ( 8 pour mille) des sportifs non dopés se situent hors normes et risquent d'être sanc- tionnés injustement. La situation s'est produite à plusieurs reprises, ce qui fragilisait la position des contrôleurs. En outre, les athlètes trouvèrent rapidement la parade en s'administrant aussi de l'épitestostérone, ce qui fut sanctionné, à partir de 1992, pour des concentrations supérieures à $150 \mathrm{ng} / \mathrm{ml}^{8}$. Toujours est-il qu'en mars 1993, le Comité International Olympique (CIO) a assoupli sa position. Désormais, la règle est la suivante : "Un taux de testostérone (T) lépitestostérone $(E)$ dans les urines supérieur à 6 constitue une infraction à moins que l'on ne puisse prouver que ce taux est dù à un état physiologique ou pathologique". L'athlète peut donc avoir recours à des tests endocriniens et à des études longitudinales pour prouver son innocence. Toute cette agitation pourrait bien prendre fin prochainement puisqu'on annonce la mise en application d'un nouveau test infaillible. Du moins, c'est la nouvelle qui nous a été donnée en grande pompe le 21 mai 1997 lors d'une réunion de la commission médicale à Monaco. Cette nouvelle méthode, mise au point il y a trois ans par des chercheurs français du CNRS, consiste à déterminer le rapport entre carbone 13 et 12 de la testostérone. L'hormone mâle endogène produite par l'individu à partir de son cholestérol contient $99 \%$ de $\mathrm{C} 12$ et $1 \%$ de $\mathrm{C} 13$ alors que la testostérone exogène, fabriquée artificiellement à partir de stéroïdes végétaux contient un peu plus de C13. S'il est incontestable que le rapport isotopique C13/C12 apparaît beaucoup plus fiable que le ratio testo/épitesto, il faut nuancer l'optimisme ambiant dans la mesure où, pour l'instant, seuls trois laboratoires au monde sont capables d'effectuer ce test et qu'il n'interviendrait de toutes façons que dans les cas litigieux. Il ne permettra pas d'attraper tous les athlètes qui continuent à consommer de la testostérone en flirtant avec cette barrière de 6 en dessous de laquelle on ne se trouve jamais inquiété. En outre, il n'empêchera pas la consommation de Gonadotrophine Chorionique (hCG), une hormone qui stimule le testicule à sécréter de la testostérone, interdite par le CIO depuis les JO de Calgary, mais toujours indécelable, elle aussi.

\section{0 : les années ÉPO}

L'idée d'accroître la teneur sanguine en oxygène pour faciliter les exercices d'endurance n'est pas franchement récente. Il y a une quinzaine d'années déjà, on sait que les cyclistes américains des Jeux de Los Angeles

${ }^{7}$ Les dernières spécialités humaines à base de nandrolone, Dynabolon $^{(1}$, Trophobolene ${ }^{(*)}$, Durabolin ${ }^{(}$et Déca-Durabolin ${ }^{(3)}$ ont été retirées des circuits de ventes au cours de l'année 1997

${ }^{8}$ En 1995 , le seuil est passé à $200 \mathrm{ng} / \mathrm{ml}$ 
ont eu recours à cette forme de dopage sanguin par laquelle on prélève un sang enrichi en globules rouges que l'on réinjecte à proximité de l'épreuve. Ces transfusions sanguines furent prohibées conjointement par le CIO et l'UCI deux ans plus tard. Mais on sait ce qu'il advint des recommandations dénuées des outils élémentaires de contrôle. Or les tests urinaires, les seuls pratiqués à l'époque, étaient muets sur ces manipulations du sang. En 1988, on institua les premiers prélèvements sanguins lors du championnat du monde de ski nordique. Au même moment, des rumeurs se mirent à circuler sur l'apparition d'une nouvelle hormone dans le sport, l'érythropoïétine, qui stimule la fabrication des globules rouges. Cette molécule que l'on apprit rapidement à désigner sous les initiales ÉPO apparut en 1990 dans le groupe $\mathrm{F}$ des substances interdites du CIO.

Mais là encore, on manque de moyens d'analyse. Si bien que l'ÉPO envahit des disciplines comme les courses de fond (où les records explosent) et l'inévitable cyclisme. Le 27 octobre 1996, le cycliste Gilles Delion raconte sur France 2:

"Les coureurs consommateurs d'ÉPO se trahissent en prenant de l'aspirine chaque soir, de manière à liquéfier le sang histoire de ne pas mourir dans la nuit"

Des cas de décès étranges surviennent effectivement dans la première partie des années 90. En 1997, la Fédération Internationale de Ski (FIS) et l'Union Internationale de Biathlon (IBU) tentent de limiter l'usage de l'érythropoïétine en imposant des seuils limites d'hémoglobine: 16,5 grammes par litre de sang chez les femmes et $18,5 \mathrm{~g} / \mathrm{l}$ chez les hommes. Le Comité International Olympique relaye ces mesures aux JO de Nagano, au mois de février 1998. Le cyclisme également, instaure au début de la saison 1997 des contrôles sanguins pour éviter les taux trop élevés de globules rouges (hématocrite supérieur à 50\%). Subtilité de vocabulaire: le coureur n'est pas suspendu mais jugé " temporairement inapte à la compétition". En clair, il ne peut pas courir pendant 15 jours.

Rappelons que, comme la plupart des seuils biologiques arbitrairement imposés par le législateur, l'hématocrite à 50 peut condamner des innocents. Les variations physiologiques de l'hématocrite d'un adulte sain varient entre 42 et $54 \%$. Mais dans la perspective toujours repoussée de la mise au point d'un test fiable de détection de l'ÉPO, les autorités sportives n'ont guère d'autre choix. Et quand bien même, ils remporteraient cette bataille, une autre se profile à l'horizon avec l'apparition d'une hémoglobine naturelle humaine de substitution qui, selon certaines sources, aurait déjà pénétré les enceintes sportives. Cette nouvelle substance aurait l'avantage sur l'ancienne d'atteindre plus facilement, grâce à sa petite taille, des tissus ou des organes auxquels le sang chargé de globules rouges parvient difficilement. Et puis, contrairement à l'ÉPO qui doit être prescrite en cure de plusieurs semaines, cette hémoglobine agirait immédiatement. Enfin, signalons que ce produit n'est toujours pas interdit et est, bien sûr, indécelable.

\section{Et les années 2000 ?}

S'ils ont subi quelques défaites au cours des trente dernières années, les dopeurs et les dopés rencontrent

\section{Les pincés}

Ces tableaux montrent le nombre d'athlètes positifs, révélés par les J0 d'été et d'hiver. Ce résultat modeste apparait avec plus d'évidence encore lorsqu'on traduit ces chiffres en pour cents. Ainsi, les contrôles aux Jeux d'hiver (5 sur 3194) montrent une efficacité de $0,13 \%$, tandis que les Jeux d'été font à peine mieux (46 sur 13018) avec $0,31 \%$. De toutes les façons, ces résultats sont très loin de la réalité du dopage.

\begin{tabular}{|lllll|}
\hline $\begin{array}{l}\text { ÉTÉ } \\
\text { Année }\end{array}$ & Ville & Nombre & Positifs & Femmes \\
\hline 1968 & Mexico (MEX) & 668 & 1 & 0 \\
1972 & Munich (RFA) & 2019 & 7 & 0 \\
1976 & Montréal (CAN) & 1166 & 11 & 0 \\
1980 & Moscou (URSS) & 1645 & 0 & 0 \\
1984 & Los Angeles (USA) & 1507 & 12 & 1 \\
1988 & Séoul (CDS) & 1598 & 10 & 0 \\
1992 & Barcelone (ESP) & 1848 & 5 & 4 \\
1996 & Atlanta (USA) & 1947 & 2 & 2 \\
TOTAL & $\mathbf{1 3 0 7 8}$ & $\mathbf{4 8}$ & $\mathbf{8}$ \\
\hline
\end{tabular}

\begin{tabular}{|lllll|}
\hline $\begin{array}{l}\text { HIVER } \\
\text { Année }\end{array}$ & Ville & Nombre & Positifs & Femmes \\
\hline 1968 & Grenoble (FRA) & 86 & 0 & 0 \\
1972 & Sapporo (JAP) & 211 & 1 & 0 \\
1976 & Innsbruck (AUT) & 290 & 2 & 1 \\
1980 & Lake Placid (USA) & 440 & 0 & 0 \\
1984 & Sarajevo (YOU) & 424 & 1 & 0 \\
1988 & Calgary (CAN) & 492 & 1 & 0 \\
1992 & Albertville (FRA) & 522 & 0 & 0 \\
1996 & Lillehammer (NOR) & 529 & 0 & 0 \\
1998 & Nagano (JAP) & 700 & 0 & 0 \\
TOTAL & $\mathbf{3 7 9 4}$ & $\mathbf{5}$ & $\mathbf{1}$ \\
\hline
\end{tabular}




\section{Les années mortes}

Pour les principales substances dopantes, on observe un temps de latence entre leur utilisation par les sportifs, et leur interdiction érigée par le CIO. Il se compte en années, parfois en décennies.

\begin{tabular}{|c|c|c|c|c|}
\hline $\begin{array}{l}\text { Substance } \\
\text { dopante }\end{array}$ & $\begin{array}{l}\begin{array}{l}\text { Année de } \\
\text { découverte }\end{array} \\
\end{array}$ & $\begin{array}{l}\mathbf{1}^{\mathrm{e} e} \text { utilisation } \\
\text { sportive }\end{array}$ & $\begin{array}{l}\text { Interdiction } \\
\text { par le CIO }\end{array}$ & $\begin{array}{l}\text { Année } \\
\text { d'identification }\end{array}$ \\
\hline Amphétamines & 1930 & 1936 & 1968 & 1968 \\
\hline Anabolisants (stéroïdes) & 1940 & 1954 & 1974 & 1976 \\
\hline Bêta-bloquants & 1958 & 1978 & 1985 & 1985 \\
\hline Coramine & $1925^{*}$ & 1929 & 1968 & 1968 \\
\hline Corticoïdes "naturels" & 1936 & 1960 & 1987 & Indécelables** \\
\hline Diurétiques & $1956^{*}$ & 1968 & 1966 & 1986 \\
\hline Erythropoïétine (EPO) & 1950 & 1987 & 1990 & Indécelable** \\
\hline Ephédrine & 1934* & 1964 & 1968 & 1968 \\
\hline Heptaminol & 1953 & 1956 & 1968 & 1968 \\
\hline Hormone de croissance (hGH) & 1944 & 1980 & 1989 & Indécelable** \\
\hline Probénécide (masquant) & $1954^{*}$ & 1976 & 1987 & 1987 \\
\hline Testostérone (hormone male) & 1935 & 1952 & 1982 & 1982 \\
\hline
\end{tabular}

* Date de mise sur le marché des médicaments français

** À ce jour, aucun seuil légal de présence urinaire n'ayant été défini, et les contrôles sanguins étant interdits aux JO, il est impossible au biologiste de conclure à l'apport exogène (médicaments)

Référence : Dopage aux Jeux Olympiques, la triche récompensée .- Jean-Pierre de Mondenard, Paris, éd. Amphora, 1996

aujourd'hui des perspectives plutôt favorables. Les progrès de la pharmacologie leur offrent sans cesse des pistes nouvelles, alors que les anciennes n'ont pas encore toutes été explorées. Les produits indécelables à leur disposition sont nombreux et, dans les prochaines années, cette pharmacopée s'enrichira à la fois de précurseurs -tels que les facteurs hypothalamiques- et d'effecteurs -au sein même de la cellule musculaire-. On sait ainsi que les effets de l'hormone de croissance sont indirects, c'est-à-dire qu'elle-même libère les véritables facteurs de croissance connus sous le nom de somatomédines. Ces derniers, véritables "maçons" des différents tissus (os, muscles, cartilages) révèlent des perspectives intéressantes. De même, certains dérivés sanguins ou apparentés prendront prochainement la relève de l'ÉPO devenue trop voyante. De plus, on progresse aussi dans la connaissance des neurotransmetteurs et on imagine l'attrait d'un médicament qui accélérerait la gestion des informations par le cerveau. Enfin, les tricheurs ont toujours la possibilité de recourir à l'hormone de croissance (GH) pour assurer des résultats. Car même si l'on connaît ce produit depuis vingt ans, on ne possède encore aucun test fiable de détection. Pour se rendre compte de son importance dans le sport, on peut seulement se baser sur des témoignages comme celui du sprinter italien Pietro Mennea qui affirme qu'il en consommait aux JO de Los Angeles en 1984. Comment expliquer un tel retard? Il est dû en partie à la complexité du problème, mais également à une mauvaise évaluation de départ. Longtemps, le CIO a minimisé l'importance de cette drogue. Par exemple, Manfred Donike (25), le grand patron de la lutte antidopage, affirmait en 1993 :

"L'efficacité de l'hormone de croissance n 'a jamais été prou- vée (...) Dans les études en double aveugle effectuées en laboratoire, rien n'indique un effet anabolisant dans le domaine sportif. Certes, ces hormones sont susceptibles d'accélérer le développement chez l'enfant en retard de croissance, mais elles restent sans effet sur des individus de taille normale".

Deux ans plus tard, le retournement de l'opinion est spectaculaire. En septembre 1995, les experts médicaux du CIO reconnaissent humblement l'importance de cette hormone (26) :

"Nous savons maintenant que la GH est une des drogues les plus utilisées dans le sport de haut niveau. Les athlètes savent tous que c'est l'agent anabolisant le plus puissant disponible, qu'il n'existe aucun moyen de détection de l'abus de cette substance et donc aucun risque de disqualification".

Le Comité International Olympique et la Communauté Européenne décident conjointement, pour rattraper le temps perdu, de consacrer plus de deux millions de dollars à l'identification du produit. Sans résultat jusqu'ici ! En fait, le seul frein à une utilisation encore plus répandue du produit est son coût prohibitif. On estime que le traitement annuel pour raison médicale varie de 35000 à 50000 francs. Dans une optique de dopage, celui-ci devrait être multiplié par 10 ! Et pour les plus pauvres? Il leur reste la possibilité de recourir à des produits meilleur marché, comme la cortisone naturelle, par exemple, toujours indécelable et qui fit la preuve de son efficacité au fil des décennies. C'est même un des dopants les plus efficaces. Lors de tests réalisés sur des chevaux, les experts toxicologues de la Société d'Encouragement ont essayé toutes sortes de molécules en vogue dans les milieux athlétiques. La plupart sont demeurées sans effet sur la performance. Seuls deux produits, dont les corticoìdes, ont amélioré de façon signifi- 
cative les résultats. Chez les hommes, il en va probablement de même. On sait que la cortisone permit à Bernard Thévenet $(27,28)$ de remporter deux Tours de France.

"J'ai été dopé à la cortisone pendant trois ans", raconte-t-il à Pierre Chany, journaliste à L'Équipe, "et ils sont nombreux dans mon cas."

Freddy Maertens l'admet d'ailleurs très volontiers et si le grand Merckx garde le silence, d'autres parlent pour lui comme Jacques Goddet (29), le patron du Tour qui, dans son ouvrage de souvenirs publié en 1991, attribue sa subite fin de carrière à la consommation de corticoïdes. Alors répétons qu'aujourd'hui encore, la prise de cortisone naturelle ne donne cours à aucune sanction ${ }^{9}$. La chose paraît même complètement illogique Si l'on songe que la substance est sanctionnée -au delà d'1 microgramme par millilitre- chez les chevaux. En 1997, une étude sur des cyclistes de haut niveau révélait même que chez $80 \%$ d'entre eux, on retrouvait des concentrations abusives de corticoïdes. Cela indique bien que le produit fait toujours l'objet de prescriptions, soit directement sous forme de cortisone, soit sous forme d'ACTH ${ }^{10}$ un produit qui augmente lui aussi les taux de corticostéroïdes endogènes dans le sang. Or, on sait pertinemment bien que cette forme de dopage ruine la santé à l'échéance d'une douzaine d'années seulement. D'ailleurs, c'est le cas de tous ces produits miracles. Les succès du moment condamnent à des fins de carrière difficiles. Un organisme malmené finit toujours par présenter la note. Les plus grandes craintes nous habitent sur les conséquences à long terme des méthodes actuelles de dopage.

Mais là, ce n'est plus de l'Histoire avec un grand $\mathrm{H}$ mais l'actualité récente des drogues de la performance.

'Les corticoïdes synthétiques n'ont été décelés officiellement qu'à partir du Tour de France 1999

10 L'ACTH (hormone peptidique corticotrophine) fit son apparition sur la liste des substances et procédés interdits en avril 1989 (CIO) et en décembre 1989 (Jeunesse et Sports). Toujours indécelable.

\section{Références}

1. Guyot-Dobes. Les hommes-phénomènes. Paris : éd. Masson, $1885: 79-82$.

2. Demole V. Médecine sportive et doping in "Contrôle médico-sportif et fatigue", recueil des travaux présentés au cours de médecine sportive tenu à Lausanne du 16 au 18 octobre 1941. Lausanne (SUT) : éd. Librairie de l'Université F. Rouge, 1941 : 183-202.

3. Schweitzer A. À l'orée de la forêt vierge. Récits et réflexions d'un médecin en Afrique Équatoriale Française. Faits se rapportant aux années 1913-1917. Paris : éd. Albin Michel, 1952 : 69-70.

4. Chany P. La fabuleuse histoire du cyclisme. Paris : éd. ODIL, $1975: 451$.

5. Sport-Digest. Sport et dynamite. Sport Digest, 1949 ; $n^{\circ} 2: 40-43$.

6. Hamelle P. Comment engager la lutte contre le "doping". Le Miroir des Sports, $1921 ; n^{\circ} 76$ : 372.

7. Novich M.M. Sport et doping. Abbotempo, Londres (GBR), $1964 ; n^{\circ} 2$ : 26-29.

8. Petit L. Causerie du docteur : l'entraînement. La Bicyclette, $1894 ; 3, \mathrm{n}^{\circ} 90: 2520-21$.

9. Petit L. Causerie du docteur : le maté. La Bicyclette, $1894 ; 3, n^{\circ} 116: 2411-12$.

10. Petit L. Causerie du docteur : la tête et les jambes. La Bicyclette, $1895 ; 4, n^{\circ} 145: 4344-47$.

11. Londres A. Les frères Pélissier et leur camarade Ville abandonnent. Le Petit Parisien, 27.06.1924.

12. Parienté R., Lagorge G. La fabuleuse histoire des Jeux olympiques. Paris : éd. ODIL, $1973 ; 65$.

13. Romain J. De l'emploi de la caféine dans le doping. Le Sport Universel illustré, $1903 ; \mathrm{n}^{\circ} 385$ : 771-72.

14. Mars R.G. [Le dopage des chevaux de course] (en anglais). Brit. Vet. J., $1958 ; n^{\circ} 288,288-294$.
15. Francis $\mathrm{Ch}$. Le piège de la vitesse. Paris : éd. Robert Laffont, 1992.

16. Berendonk B. [Dossier dopage. De la recherche à la fraude] (en allemand). Berlin Heidelbert (ALL) : éd. Springer-Verlag, 1991.

17. Dubin C.L. Commission d'enquête sur le recours aux drogues et autres pratiques interdites pour améliorer la performance athlétique. Ottawa (CAN) : éd. Centre d'édition du gouvernement du Canada. Approvisionnements et services Canada, $1990: 380$.

18. L'Équipe. 18.08.1988.

19. L'Équipe. 21.06.1989.

20. Brera G. Le géant et la lime. Vicenza (ITA) : éd. Campagnolo, 1995 : 70.

21. Herzog M. Annapurna premier 8000 . Paris : éd. Arthaud, $1968: 100$ et 190 .

22. Dyhrenfurth G.O. L'Himalaya troisième pôle. Paris : éd. Payot, $1953: 147$.

23. Terray L. Les conquérants de l'inutile. Paris, éd. Gallimard, $1961: 456$.

24. de Mondenard J.-P., Chevalier B. Le dossier noir du dopage. Paris : éd. Hachette, 1981: 237.

25. Donike M. Dopage : il faut protéger les athlètes (propos recueillis par Gilles Goetghebuer). Sport et Vie, $1993 ; 4$, $n^{\circ} 17: 57-63$.

26. Cabut S. La chasse au dopage à l'hormone de croissance. Impact Médecin Quotidien, 03.10.1995 ; Document CIO, 09.1995.

27. Chany P. 17 coureurs morts de crise cardiaque. ParisMatch, $1978 ; n^{\circ} 1539$ : 93-112.

28. Chany P. L'angoissant combat de Bernard Thévenet. Vélo, $1978 ; n^{\circ} 125: 3-5$.

29. Goddet J. L'équipée belle. Paris : éd. Robert Laffont / Stock, $1991: 350$. 